\title{
La propagation de la sécurité : l'Europe et la schengenisation de la Politique de voisinage
}

Spreading security: Europe and the Schengenisation of the Neighbourhood Policy

Ruben Zaiotti

\section{OpenEdition}

Journals

Édition électronique

URL : http://journals.openedition.org/conflits/2471

DOI : $10.4000 /$ conflits. 2471

ISSN : $1777-5345$

Éditeur :

CCLS - Centre d'études sur les conflits lilberté et sécurité, L'Harmattan

Édition imprimée

Date de publication : 17 septembre 2007

Pagination : 61-76

ISBN : 978-2-296-03458-7

ISSN : 1157-996X

Référence électronique

Ruben Zaiotti, « La propagation de la sécurité : I'Europe et la schengenisation de la Politique de voisinage », Cultures \& Conflits [En ligne], 66 | été 2007, mis en ligne le 09 juillet 2007, consulté le 30 mars 2021. URL : http://journals.openedition.org/conflits/2471 ; DOI : https://doi.org/10.4000/ conflits. 2471 


\section{La propagation de la sécurité : I'Europe et la schengenisation de la Politique de voisinage .}

\section{Ruben ZAIOTTI}

Ruben Zaiotti est doctorant au département de sciences politiques de l'université de Toronto. Son principal champ de recherche est la sécurité internationale, plus particulièrement en Europe. Il a récemment publié des articles dans The Journal of European Politics and Society et International Journal of Refugee Law. Il est actuellement rédacteur en chef de la revne Journal of International Law and International Relations.

\section{La PEV et le sens de la sécurité}

T a sécurité est un élément central de la Politique européenne de voisinage $\mathcal{L}(\mathrm{PEV})$, une initiative récente de l'Union européenne à l'attention des pays qui, depuis la dernière vague d'élargissement de l'Union, se trouvent désormais aux frontières extérieures de l'Europe ${ }^{1}$. L'objectif affiché de la PEV est en fait d'établir, autour des bordures de l'Europe, un "cercle d'amis" - caractérisé par des relations étroites et pacifiques fondées sur la coopération 2 ». En d'autres termes, l'Europe rêve de la création d'une «communauté de sécurité ${ }^{3}$ » pan régionale. Pour y parvenir, la PEV s'est dotée d'une série de mesures ayant pour but de protéger l'UE et ses voisins de menaces communes comme le terrorisme, l'immigration clandestine et le trafic de stupéfiants. Ces mesures sont toutefois présentées comme corollaires à la principale motivation qui voudrait que la PEV garantisse que cette communauté de sécurité devienne une réalité, notamment la promesse faite aux voisins de leur offrir un accès élargi au marché commun.

* Cet article a été traduit de l’anglais par Miriam Perier.

1. Ces pays incluent la Biélorussie, la Moldavie et l’Ukraine en Europe de l'Est; l'Arménie, l'Azerbaijan et la Géorgie dans le Sud-Caucase ; l'Algérie, l'Egypte, Israël, la Jordanie, le Liban, la Libye, le Maroc, l’Autorité palestinienne, la Syrie et la Tunisie en région méditerranéenne du Sud. Bien qu'il s'agisse aussi d'un voisin, les relations avec la Russie sont traitées séparément, via le « partenariat stratégique».

2. Commission européenne, «Wider Europe - neighbourhood: a new framework for relations with our Eastern and Southern neighbours », COM (2003) 104 final, 2003, p. 2.

3. Adler E., Barnett M., (eds), Security Communities, Cambridge, Cambridge University Press, 1998. 
Selon ses défenseurs, cette initiative devrait être conduite dans un esprit de collaboration et de réciprocité. Comme le disait Romano Prodi, l'ancien président de la Commission européenne :

«Mon but est de leur donner [aux voisins de l'UE] certaines formes d'encouragement, en injectant une nouvelle dynamique dans des processus déjà existants et en développant un partenariat ouvert et évolutif. C’est ce que nous appelons notre politique de proximité : une politique fondée sur des profits et des obligations mutuels, ce qui constitue une contribution substantielle de l'UE à la gouvernance mondiale 4 ».

Au premier abord, la PEV semble constituer un développement prometteur de la politique étrangère de l'UE. Elle représente une approche globale et progressive des conséquences de l'élargissement et des nouveaux défis et opportunités apportés par les nouveaux voisins. Elle est fondée sur une relation de quiproquo entre l'UE et ses voisins, qui devrait avoir des conséquences positives pour les deux parties, et semble, en ce sens, la dernière application en date du modèle européen de politique étrangère « civile » 5 .

Toutefois, si nous observons de plus près la manière dont la sécurité a été intégrée à la PEV, nous constatons que ce projet est moins bienveillant que ses défenseurs veulent bien le laisser entendre. Plutôt que d'encourager la stabilité et la prospérité autour de l'Union, les questions de sécurité semblent prendre le pas sur la possibilité d'atteindre ces objectifs. Par ailleurs, la PEV semble moins porter un véritable esprit de partenariat que projeter une vision très euro-centrée de ce que la sécurité régionale implique, une vision que l'on impose - certes avec « douceur »- au proche étranger européen. On a donc le risque que la PEV reproduise - voire renforce - certaines des «divisions» européennes ${ }^{6}$ que le projet cherche justement à dissiper.

Cet article vise à mettre en avant les éléments sécuritaires et euro-centriques de la $\mathrm{PEV}$ et à proposer une approche critique de ses implications pour les relations entre l'UE et ses voisins ${ }^{7}$. Nous souhaitons contribuer au débat

4 . Prodi R., «A wider Europe - A proximity policy as the key to stability », discours prononcé lors du 6e ECSA-World Conference, Bruxelle, 5-6 décembre 2002. Nous traduisons, nous soulignons.

5. Voir Dûchene F., «Europe's role in world peace », in Mayne R. (ed.), European Tomorrow: Sixteen Europeans Look Abead, London, Fontana, 1972, pp. 32-47; Rosencrance R., « The European Union: A new type of international actor », in Zielonka J. (ed), Paradoxes of European Foreign Policy, The Hague, Kluwer Law International, 1998, pp. 15-23.

6. COM (2003) 104 final, 2003, op. cit., p. 1.

7. Sur la dimension sécuritaire de la PEV, voir Guild E., "What is a neighbour? Examining the EU Neighbourhood Policy from the perspective of movement of persons ", papier présenté au Forum Western NIS for Refugee-Assisting NGOs, Yalta, $1^{\text {er }}-3$ juin 2005 ; sur l'européanocentrisme, voir Johansson-Nogués E., " A "ring of friends"? The implications of the European Neighbourhood Policy for the Mediterranean ", Mediterranean Politics, vol. 2, n 9 , 2004, pp. 240-247; sur la PEV en général voir Dannreuther R., « Developing the alternative to enlar- 
sur la politique de voisinage en montrant que ces éléments sont fortement interconnectés et se renforcent mutuellement. Nous explorerons également les possibles raisons de la tournure sécuritaire spécifique de cette initiative européenne. Dans la littérature existante sur la PEV, ses défauts et lacunes ont été identifiés et rattachés aux conséquences du processus d'élargissement ${ }^{8}$, et en particulier à la peur de l'instabilité aux portes de l'Europe. Ces analyses n'ont toutefois pas expliqué de manière satisfaisante les racines des tensions intrinsèques de la $\mathrm{PEV}$ que nous avons précédemment décrites. Nous proposons donc une approche sociologique fondée sur la notion de «culture Schengen de la sécurité » (du nom du régime d'origine de cette culture). Nous considérons plus particulièrement que le rôle de la sécurité dans la PEV est un résultat de la consolidation récente de cette culture et de sa « diffusion » au sein de plusieurs champs de politiques de l'UE qui ont affecté la PEV. Nous souhaitons montrer que la schengenisation de la PEV ne s'est pas faite «à l'insu » des principaux acteurs impliqués dans son élaboration (notamment la Commission et le Conseil européens), mais qu'elle a été soutenue et reproduite via leurs pratiques et leurs interactions dans le processus d'élaboration de cette politique.

\section{La PEV et la sécurité : pour une analyse critique}

Au tournant du millénaire, l'élargissement imminent de l'UE a soulevé des questions sur l'avenir de pays qui, "distants» avant l'élargissement, allaient dorénavant se trouver aux frontières de l'UE. Le souvenir des Balkans et le spectre d'une instabilité aux portes de l'Europe étaient encore vivaces dans les esprits des responsables politiques européens, et c'est cet esprit qui a prévalu lors de l'élaboration de la PEV. C'est en 2002 que les institutions et les gouvernements de l'UE ont commencé à aborder le débat des relations à venir

gement: the European Neighbourhood Policy ", European Foreign Affairs Review, vol. 11, $\mathrm{n}^{\circ} 2$, 2006, pp. 183-201; Kelley J., « New wine in old wineskins: promoting political reforms through the new European Neighbourhood Policy ", Journal of Common Market Studies, vol. 44, n`1, 2006, pp. 29-55 ; Del Sarto R.A., Schumacher T., « From EMP to ENP: what's at stake with the European Neighbourhood Policy towards the Southern Mediterimplications of the European Neighbourhood Policy ", European Foreign Affairs Review, vol. 10, n ${ }^{\circ}, 2005$, pp. 1-16. ; Goujon A., "L'Europe élargie en quête d'identité : légitimation et politisation de la politique européenne de voisinage », Politique européenne, n¹5, 2005, pp. 137-163 ; Lynch D., "The challenges of the European Neighbourhood Policy - the security dimension of the European Neighbourhood Policy ", International Spectator, vol. 40, n¹, 2005. ; Smith K.E., «The outsiders: the European Neighbourhood Policy », International Affairs, vol. 81, n4, juillet 2005, pp. 757-773 ; Tocci N., " The challenges of the European Neighbourhood Policy. Does the ENP respond to the EU's post-enlargement challenges? ", International Spectator, vol. 40, ${ }^{\circ} 1$, 2005; Balfour R., Rotta A., «Beyond enlargement. The European Neighbourhood Policy and its tools ", The International Spectator, vol. 40, n 1,2005 , pp. 720 ; Lavenex S., "EU external governance in "Wider Europe" ", Journal of European Public Policy, vol. 11, n4, août 2004, pp. 680-700; Comelli M., "The challenges of the European Neighbourhood Policy ", The International Spectator, vol. 39, n³ 3 , juillet-septembre 2004, pp. 97-110; Pardo S., "Europe of many circles: European Neighbourhood Policy», Geopolitics, vol. 9, n³, 2004.

8. Voir Del Sarto R.A., Schumacher T., op. cit., pp. 17-38 ; Kelley J., op. cit., pp. 29-55. 
avec les futurs voisins de l'UE 9. Ces discussions préliminaires ont donc précédé la présentation en mars 2003, par la Commission européenne, d'un plan établissant les grands principes de ce que l'on appelait à l'époque «l'initiative de l'Europe élargie 10 ». Un an plus tard, le plan fut complété par un Document d'orientation 11 établissant le cadre géographique et la méthodologie de la mise en œuvre de cette nouvelle politique.

La Commission détaille dans ces documents les étapes que les voisins devraient suivre pour tirer parti de cet accès direct au marché commun européen. L'approche proposée est " différenciée », mais fondée sur des critères de référence communs (benchmarks). Les particularités propres des pays concernés et la nature complexe des questions traitées ont poussé la Commission à proposer un cadre flexible à cette politique - à l'inverse du modèle « uniforme » utilisé pour l'élargissement dans les années 1990. L'établissement des priorités et le calendrier de leur mise en ouvre dépendraient d'une variété de critères, comprenant la localisation géographique, le contexte politique et économique, et le statut des relations avec l'UE et d'autres pays ${ }^{12}$. La réalisation ou non des objectifs de l'initiative serait mesurée en fonction d'une série de critères prédéfinis, à savoir le progrès dans l'acquisition de valeurs partagées, la mise en œuvre effective de réformes politiques, économiques et institutionnelles, ainsi que l'alignement législatif sur les acquis de l'UE. Le domaine de la Justice et des Affaires intérieures (JAI) apparaît comme une priorité. Cette section spéciale promeut une coopération rapprochée dans des domaines tels que la gestion des frontières, l'immigration, la lutte contre le terrorisme, le trafic d'êtres humains, de stupéfiants et d'armes, le crime organisé, le blanchiment d'argent et les crimes financiers et économiques ${ }^{13}$.

La dimension opérationnelle de la PEV est définie par une série de plans d'action, des documents de politiques décrivant les cibles et les références stratégiques globales. Ceux-ci comportent un ensemble de priorités approuvées

9. La première présentation officielle de cette stratégie est apparue en janvier 2002 sous la forme d'une lettre du ministre britannique des Affaires étrangères, Jack Straw, à la présidence espagnole. Ce document prévoyait une approche globale vers certains des voisins européens (la Biélorussie, la Moldavie et l'Ukraine), une approche similaire à celle adoptée par l'UE envers les Balkans occidentaux (c'est-à-dire une plus forte intégration), mais sans la « carotte » d'une adhésion future à l'UE. La lettre Straw fut suivie d'une initiative suédoise, la lettre LindhPagrotsky, qui suggérait un élargissement géographique à la politique (son expansion vers les pays du Sud-Méditerranée) et une plus grande importance placée sur le libre échange et le développement économique. (Foreign and Commonwealth Office, Lettre de Jack Straw à Josep Piqué, Londres, 28 janvier 2002; Regeringskansliet, Lettre de Ms. Anna Lindh [Ministre des Affaires étrangères] et M. Leif Pagrotsky [Ministre du Commerce international] à Josep Piqué, Stockholm, 8 mars 2002).

10. Le plan était inclus dans une communication au Conseil et au Parlement européens, intitulé Wider Europe - Neighbourhood: A New Framework for Relations with our Eastern and Southern Neighbours, COM (2003) 104 final.

11. Communication de la Commission, "European Neighbourhood Policy: strategy paper ", COM (2004) 373 final.

12. Del Sarto et Schumacher qualifient cette approche de "bilatéralisme différencié ", voir Del Sarto R.A., Schumacher T., op. cit, p. 5.

13. COM (2003) 104 final, op. cit., pp. 11-12. 
collectivement dans des domaines spécifiques tels que la Justice et les Affaires intérieures ${ }^{14}$. Une fois approuvés, ces plans d'action devront supplanter les accords bilatéraux préexistants afin de devenir le principal cadre politique des relations de l'UE avec ses voisins sur le moyen terme, et former les fondements à de nouveaux « accords de voisinage » entre les deux parties 15 .

La référence au domaine de la Justice et des Affaires intérieures comme domaine central est digne d'intérêt car il s'agit d'une nouvelle tendance politique dans les relations entre les deux côtés, même si des accords bilatéraux antérieurs comportaient des éléments de sécurité « intérieure ». En 1995 par exemple, l'UE a inséré une « clause standard » dans plusieurs accords de coopération avec des pays européens de l'Est ${ }^{16}$. Toutefois, avant le lancement de la PEV, les questions sécuritaires ne jouaient qu'un rôle assez marginal dans la coopération entre l'UE et ses voisins et il n'existait pas de plan détaillé de mise en œuvre des provisions ${ }^{17}$.

Les questions sécuritaires étaient très présentes dans la PEV dès ses premières formulations. Ainsi, Jack Straw, dans sa lettre à la présidence espagnole, dresse un portrait assez pessimiste des voisins :

«L'Ukraine et la Biélorussie seront aux frontières de l'UE dans trois ans - avec tous les problèmes attenants de criminalité transfrontalière, de trafic et d'immigration clandestine. La Moldavie ne sera un voisin de l'Union que plus tard $[. .$.$] mais elle est déjà$ confrontée à une pauvreté écrasante, d'importants problèmes sociaux et une émigration massive 18 ».

Cette inquiétude des voisins et de leur effet potentiellement déstabilisant sur le continent est l'une des idées maîtresses sous-jacentes à la PEV. En effet, bien que cela reste discret, les questions de sécurité sont entrées dans les communications de la Commission. On y reconnaît que tous les partis ont intérêt à veiller à ce que les frontières communes ne se transforment pas en barrières au commerce et aux autres types d'échanges légaux. En même temps, une sécurité renforcée est l'un des pré-requis au succès de cette initiative :

14. Les autres domaines que la Commission indique sont le dialogue et la réforme politique, la réforme et le développement économiques et sociaux, les questions de régulations et de commerce et les contacts interpersonnels (COM (2003) 104 final).

15 . Des plans d'action préliminaires avec un premier groupe de partenaires de la PEV ont été présentés par la Commission en décembre 2004. Au printemps 2007, la plupart des plans d'action ont été officiellement adoptés par l'UE.

16. Cette clause exige des pays signataires qu'ils rappellent leur population si la demande leur est faite.

17. Les plans d'action pour la Justice et Affaires intérieures avec la Russie et l'Ukraine sont deux exceptions notables, bien qu'ils aient été conclus peu avant les premières discussions sur la PEV (avril 2000 et décembre 2001, respectivement ; Potemkina O.I., "EU-Russia cooperation in Justice and Home Affairs in the context of enlargement ", IEE Document n'23, Louvain, Institut d'études européennes, université catholique de Louvain, 2002, p. 7).

18. Foreign \& Commonwealth Office, op. cit. Notre traduction. Voir l'annexe n ${ }^{\circ} 6$ à ce numéro. 
«Il conviendra d'adopter des démarches conjointes pour lutter ensemble contre les menaces à la sécurité commune découlant du caractère transfrontalier des risques écologiques et nucléaires, des maladies transmissibles, de l'immigration clandestine, des trafics, de la criminalité organisée ou encore des réseaux terroristes ${ }^{19}$ ».

Mais ces dernières mesures dépassent le simple caractère compensatoire. La PEV exige des voisins européens qu'ils renforcent les contrôles afin de prévenir les menaces avant que celles-ci n'atteignent le cœur du continent. En termes de gestion des frontières, le rôle des voisins est donc d'interdire l'entrée aux citoyens des pays tiers que les Etats-membres considèrent comme des personae non gratae. Les voisins devraient donc devenir des zones-tampons entre l'UE et ce qui est considéré comme les sources originelles des menaces potentielles (par exemple l'Afrique sub-saharienne pour l'immigration clandestine).

Une des conséquences de la sécurisation de la relation entre l'UE et ses voisins est qu'au nom d'une soi-disant « amitié » future avec l'UE, ces pays voisins prennent le risque, non seulement de perdre le soutien de leur population puisque les gouvernements auront à prendre des mesures coercitives à l'encontre de leurs citoyens -, mais aussi d'attenter aux bonnes relations qu'ils peuvent entretenir avec des pays tiers. Certaines provisions de la PEV exigent également que les voisins intensifient les contrôles aux frontières européennes comme aux frontières extérieures. Cette obligation renforce l'idée que les voisins constituent une menace potentielle et qu'ils font donc partie intégrante du problème que la PEV tente de « régler ». La sécurisation des contrôles aux frontières pourrait donc aller à l'encontre de l'un des objectifs annoncés de cette initiative, celui de renforcer et de concrétiser davantage le dialogue politique entre l'Europe et ses voisins. Une des conséquences inattendue de la PEV pourrait donc être d'accroître l'instabilité et le conflit, au lieu d'approfondir la solidarité.

Ces implications - problématiques - des mesures sécuritaires sont évidentes lorsque l'on observe la section dédiée aux mouvements de populations. L'une des principales incitations de la PEV est d'offrir la possibilité aux citoyens des pays avoisinants d'entrer plus facilement dans l'Union (la libre circulation des individus apparaît comme l'une des « quatre libertés » qui définissent le marché commun). Il existe en effet une référence à des possibles "perspectives en matière de migration légale et de circulation de personnes 20 " dans la communication de mars 2003. Mais, si nous observons de près ces propositions, la générosité apparente s'estompe ${ }^{21}$. Certains pays bénéficient de

19. COM (2003), op. cit., p. 3.

20. COM (2003), op. cit., p. 3 et p. 7.

21. A ce sujet, voir Guild E., "What is a neighbour? Examining the EU Neighbourhood Policy from the perspective of movement of persons », op. cit. 
procédures simplifiées en termes de visas de courts séjours, mais cela ne constitue qu'une petite minorité 22. Pour la majorité, il n'y a pas d'expansion des rares provisions existant déjà dans les accords bilatéraux entre l'UE et ses voisins. Et justement, la référence à l'accès aux « quatre libertés » de l'UE, comprenant la liberté de mouvement, est reléguée au Document d'orientation, et se voit remplacée par des termes bien plus vagues 23 .

La nature problématique des provisions sécuritaires de la PEV apparait du fait que ce qui est présenté comme un projet commun semble en réalité refléter une vision euro-centrée de l'initiative et de ses priorités. La PEV annonce les problèmes comme étant collectifs et leur résolution, en conséquence, collective. Dans la communication de mars 2003, la Commission déclare que l'UE « et chacun de ses voisins partagent de nombreux intérêts ${ }^{24}$ ». L'existence de ces intérêts communs exige à son tour une coopération des deux côtés. L'omniprésence des termes «mutual », «joint » et «shared 25 témoigne de l'attachement des défenseurs de la PEV à la nature collective du projet. Dans les communications de la Commission de 2003 et 2004, ces termes apparaissent jusqu'à cinquante-cinq fois...

Mais jusqu'à quel point ces problèmes et solutions sont-ils réellement partagés ? Un premier élément nous indique qu'ils ne le sont pas : l'intitulé même de ce projet : "Politique européenne de voisinage ». L'utilisation du terme "européenne » indique clairement la zone couverte par l'UE et ses Etats-membres ${ }^{26}$. Ce terme indique en réalité non seulement la portée mais aussi la "propriété » de cette initiative. Puisque l'UE est la partie ayant le plus d'intérêts en jeu dans le projet, elle peut établir l'agenda, y compris ce qui constituera les principales priorités "partagées ». Considérés sous cet angle, les intérêts officiellement partagés se résument finalement aux intérêts de l'UE, et en particulier le renforcement de sa sécurité. Cela parait évident si l'on compare la PEV à la «Stratégie européenne de sécurité », dans laquelle l'UE annonce son approche des défis du nouveau millénaire en termes de sécurité ${ }^{27}$. L'UE et ses Etats-membres déclarent dans le Document d'orientation - publié au moment du lancement officiel de la PEV - que la distinc-

22. Il ne faut pas oublier qu'à l'heure actuelle, tous les citoyens des pays membres de la PEV (à l'exception d'Israël) doivent obtenir un visa avant de pouvoir entrer dans la zone Schengen.

23. L'UE ne peut offrir que des "mesures préparant les partenaires à une participation progressive au marché intérieur [...] » (Document d'orientation, p. 2).

24 .p. 3.

25. NDT : Nous avons préféré laisser les termes en anglais. Leur traduction française serait: « mutuel », « commun » et " partagé ». Ces trois termes apparaissent trente-huit fois dans la communication de mars 2003 de la Commission. L'analyse reste donc valable dans les deux langues.

26. La nature euro-centrée du projet était encore plus apparente dans la version originale du texte, qui, comme nous l'avons déjà mentionné, était appelé « Europe élargie ».

27 . «Une Europe sûre dans un monde meilleur. Stratégie européenne de sécurité », Bruxelles, 12 décembre 2003. Pour un commentaire critique, voir Toje A., « The 2003 European security strategy: A critical appraisal », European Foreign Affairs Review, vol. 10, n¹, 2005, pp. 117-134. 
tion entre la sécurité «hard» (militaire) et «soft» (non militaire) est brouillée et qu'une meilleure coordination entre ces deux champs est nécessaire. Ce document met également en avant l'importance d'un voisinage pacifique et stable pour l'UE :

«Il est dans l'intérêt de l'Europe que les pays situés à ses frontières soient bien gouvernés. Les voisins engagés dans des conflits violents, les Etats faibles où la criminalité organisée se répand, les sociétés défaillantes ou une croissance démographique explosive aux frontières de l'Europe constituent pour elle autant de problèmes. Notre tâche doit être de promouvoir, à l'Est de l'Union européenne et aux frontières du bassin méditerranéen, un ensemble de pays bien gouvernés avec lesquels nous pourrons avoir des relations étroites, fondées sur la coopération 28 ».

Dans ses références aux voisins de l'UE, le Document d'orientation utilise un langage similaire, voire identique presque mot pour mot à celui des communications de la Commission. Si la PEV n'est pas explicitement mentionnée dans le texte, la vision exprimée dans ce dernier coïncide globalement avec le programme de sécurité plus large de l'UE.

La nature euro-centrée de la PEV apparaît également clairement dans la manière dont est décrite la relation entre l'UE et ses voisins. Bien que les pays participant au projet soient souvent qualifiés de "partenaires ", le terme "partenariat " n'est jamais utilisé ${ }^{29}$. L'idée même de créer un "cercle d'amis" autour de l'Europe suggère que l'objectif n'est pas la mise en place d'un système « horizontal » dans lequel chaque acteur interagit à un niveau égal, mais plutôt un système « concentrique » dont l'Europe constitue l'épicentre et les voisins les différents rayons. Cet arrangement renforce plus qu'il ne remet en cause les disparités «qui existent vraiment » entre les deux camps, en termes de population, de richesse et de pouvoir.

La Commission a essayé de contrer cette perception d'une Europe qui imposerait son calendrier aux voisins. Dans le Document d'orientation de 2004, elle aborde la question de «l'appropriation commune» :

28. Solana Javier, Une Europe sûre dans un monde meilleur. Stratégie européenne de sécurité, Document adopté par les chefs d'Etat et de gouvernement réunis en Conseil européen à Bruxelles, 12 septembre 2003, p. 15. Disponible en ligne sur : www.iss-eu.org/solana/solanaf.pdf

29. Le terme est réservé à la Russie. Du fait de la participation de Moscou à l'initiative, le nom original du principal instrument financier de la PEV fut changé de «Instrument européen de voisinage » à «Instrument européen de voisinage et de partenariat » (IEVP). Pour une analyse du rôle de la Russie dans la PEV, voir Tassinari F., " The challenges of the European Neighbourhood Policy - A riddle inside an enigma: unwrapping the EU-Russia strategic partnership' », International Spectator, vol. 40, n ${ }^{\circ}, 2005$. 
«L'appropriation commune du processus, fondée sur la prise en compte de valeurs et d'intérêts communs, est essentielle. L'UE ne cherche pas à imposer des priorités ou des conditions à ses partenaires. [...] Il ne saurait être question de demander aux partenaires d'accepter un ensemble préétabli de priorités. Celles-ci seront définies d'un commun accord et différeront donc d'un pays à l'autre 30 ».

Cet aspect est réapparu dans des documents plus récents ${ }^{31}$. Mais cette qualification n'enlève toutefois pas le sentiment que la PEV est un projet asymétrique, allant du haut vers le bas.

Cette politique semble finalement envoyer des signaux contradictoires avec, d'un côté, la promesse d'un accès élargi à l'UE et un partenariat équitable et de l'autre, une emphase sans précédent sur la sécurité, qui en pratique limite non seulement la capacité de l'UE à répondre aux attentes générées par son offre, mais engendre plus de restrictions et d'obligations onéreuses imposées aux voisins. En même temps, le contenu comme la forme de l'initiative renforcent l'asymétrie qui caractérise les deux partis impliqués. Certains des éléments qui définissent la PEV vont clairement à l'encontre des objectifs annoncés (éviter de créer de nouvelles lignes de division sur le continent).

\section{La sécurité et la schengenisation de la PEV}

Comment pouvons-nous donner un sens à la sécurisation et à la nature euro-centrée de la PEV ? Pourquoi ces éléments sont-ils devenus prépondérants ? Nous suggérons une analyse sociologique qui conceptualise les dynamiques caractérisant la prise de décisions européennes, en partant de pré-notions et de pratiques routinisées émergeant de l'interaction entre les principaux responsables politiques de la région ${ }^{32}$. Dans la lignée d'Ann Swidler, nous définissons l'interaction entre les assomptions de fond et les pratiques routinisées partagées par un groupe d'individus dans un lieu et une période donnés comme une « culture 33 ». Les " assomptions de fond » sont des structures cognitives intersubjectives auxquelles se réfèrent les membres d'une communauté donnée lorsqu'ils interprètent la réalité dans laquelle ils se trouvent et lorsqu'ils décident de l'action à entreprendre dans une circonstance donnée. Ces hypothèses deviennent pertinentes lorsque les membres de la communauté les intègrent dans leurs pratiques quotidiennes (la culture est une «boîte à outils » utilisée

30. European Commission, «European Neighbourhood Policy: strategy paper », COM (2004) 373 final, 2004 , p. 8.

31. Voir par exemple la communication de la Commission relative au «Renforcement de la politique européenne de voisinage », COM (2006) 726 final.

32. Pour une lecture des approches sociologiques de l'étude de l'Europe et de l'UE, voir Christiansen T., Joergensen K., Wiener A. (eds), "Constructivism in European studies », European Journal of Public Policy, ${ }^{\circ}$ 6, 1999.

33. Swidler A., "Culture in action: symbols and strategies ", American Sociological Review, $\mathrm{n}^{\circ}$ 51, 1986. 
pour répondre à un problème ou une situation donnés ${ }^{34}$ ). Puis, ces assomptions sont reproduites et maintenues dans le temps grâce à ces pratiques - comprises comme des modèles d'activités sociales identifiables et relativement stables dans le temps ${ }^{35}$. Les assomptions de fond et les pratiques constituent donc les deux faces d'une même pièce (la pièce étant ici la culture) ${ }^{36}$.

Nous considérons que cette interaction entre les assomptions de fond et les pratiques routinisées caractérise les dynamiques politiques qui ont mené à la création de la PEV, en tant que nouvelle politique de l'UE vis-à-vis de ses voisins. La configuration particulière de ces assomptions et pratiques vient de ce que nous appelons « la culture Shengen de la sécurité », dont l'origine remonte à l'Europe continentale du milieu des années 1980. Son émergence est associée à l'accord signé par un groupe de pays européens (France, Allemagne et Benelux dans la ville de Schengen) - prévoyant de renoncer progressivement aux contrôles aux frontières en Europe continentale ${ }^{37}$. Cet accord repose sur un positionnement central de la sécurité dans les priorités politiques, la mise en commun de la souveraineté entre gouvernements nationaux perçue comme le meilleur moyen de protéger l'Europe des menaces intérieures et extérieures, un pragmatisme et une flexibilité comme approche privilégiée dans le processus de prise de décision, et enfin une suspicion et une méfiance dans les relations avec les pays tiers. La communauté qui partage ces idées est composée de responsables politiques européens, tant aux niveaux nationaux que supranationaux (chefs d'Etat, ministres, représentants nationaux en poste à l'UE, certains officiels du Secrétariat du Conseil et de la Commission européenne). Les assomptions de cette communauté ont été traduites dans une série de pratiques qui ont caractérisé les politiques du département Justice et Affaires intérieures (JAI) de l'UE ces dernières années. Elles incluent la «transgouvernementalisation 38 » du domaine JAI par la création d'un mécanisme européen de prise de décision cogéré par la Commission européenne et le Conseil européen ; la promotion agressive de mesures restrictives pour faire face à des menaces potentielles et leur source ${ }^{39}$; et une pression sur les pays tiers pour qu'ils améliorent la sécurité, particulièrement à leurs frontières avec l’UE.

34. Ibid.

35. Schatzi, K., «Introduction », in Cetina K., von Savigny E., Schatzi K., The Practice Turn in Contemporary Theory, New York, Routledge, 2001.

36. Pour un développement du concept de «culture » et son application au cas européen, voir Zaiotti R., «Revisiting Schengen: Europe and the emergence of a new "culture of border control" ", Perspectives on European Politics and Society, vol. 8, n¹, 2007.

37. Pour une analyse de la naissance du régime Schengen et de son évolution, voir Hreblay V., Les Accord de Schengen : origine, fonctionnement, avenir, Bruxelles, Bruylant, 1998.

38. Wallace H., "The institutional setting: five variations on a theme ", in Wallace H., Wallace W. (eds.), Policy-Making in the European Union, Oxford, Oxford University Press, $4^{e}$ éd., 2000.

39. Les moyens utilisés pour mettre en œuvre ces mesures qui ont souvent été qualifiées de «politiques télécommandées» ( remote control» policies, voir Lahav G., Guiraudon V., "Comparative perspectives on border control: away from the border and outside the state ", in Andreas P., Snyder T. (eds), The Wall around the West: State Borders and Immigration Controls in North America and Europe, Lanham, Rowman \& Littlefield, 2000) incluent les régimes de visas, les sanctions aux entreprises de transport, et les politiques d'interdiction. 
Il est facile d'observer des parallèles entre les éléments qui caractérisent la culture de Schengen et ceux de la PEV. Cette dernière donne l'impression que certaines politiques sécuritaires adoptées dans le contexte de la culture Schengen ont simplement été «réemballées 40 » puis «livrées》 aux pays voisins. Néanmoins, ce qui caractérise la relation entre la PEV et la culture Schengen ne se résume pas simplement à une série de correspondances. Nous considérons que la culture Schengen s'est en réalité « propagée » dans la PEV, conséquence inattendue de sa consolidation. Comme nous l'avons mentionné précédemment, une culture évolue avec le temps et ce processus de maturation implique l'internalisation des assomptions d'une culture et la routinisation de ses pratiques ${ }^{41}$. La maturation d'une culture se mesure à l'aune du degré d'institutionalisation (la formalisation d'un corpus de notions et de pratiques spécifiques) atteint et du degré de diffusion auprès de nouveaux membres et de champs fonctionnels. Les deux processus ont marqué la trajectoire récente de la culture Schengen. Lorsque cette dernière est apparue, dans les années 1980, son institutionnalisation était «faible » et ses structures, pour la plupart informelles et en dehors du cadre européen (Communauté européenne, à l'époque). Lorsque les acquis Schengen furent incorporés à l'UE (avec l'entrée en vigueur du traité d'Amsterdam en 1999), Schengen est devenu le cadre officiel définissant le domaine JAI de l'Union. L'adhésion à cette culture est progressivement passée d'un petit groupe de pays à presque toute l'UE (à l'exception notable du Royaume-Uni et de l'Irlande), plus certains pays non membres de l'UE comme la Norvège, l'Islande et la Suisse. De plus, des préocuppations « internes » étroites autour de la question du contrôle des frontières, les hypothèses et les pratiques de Schengen ont atteint d'autres champs politiques, dont les affaires étrangères ${ }^{42}$.

La maturation de Schengen a eu lieu alors que la PEV était en cours d'élaboration, mais la culture Schengen a représenté bien plus qu'un simple environnement, elle a, selon nous, directement influencé la PEV. Comment cela a-t-il été possible ? Comme nous l'avons introduit plus tôt, nous considérons la culture comme un phénomène collectif qui existe grâce aux conséquences inattendues des interactions perpétuelles des acteurs entre eux et avec l'environnement normatif ou le «champ ${ }^{43}$ » dans lequel ils se trouvent, plus qu'elle n'est le résul-

40. Voir Guild E., "What is a neighbour? Examining the EU neighbourhood policy from the perspective of movement of persons ", op. cit.

41. Swidler A., op. cit., pp. 279-281.

42. Le traité d'Amsterdam a accentué la dimension extérieure de la politique de sécurité intérieure de l'Union. La coopération avec ses voisins «proches» ou «lointains » et avec les organismes internationaux en termes de JAI était en réalité considérée comme un composant essentiel du projet - introduit avec Amsterdam - de créer une «zone de liberté, sécurité et justice » au sein de l'Europe. Voir Monar J., "The dynamics of Justice and Home Affairs: laboratories, driving factors and costs ", Journal of Common Market Studies, vol. 39, n 4 , 2001. Voir infra. Sur le lien entre Schengen et les voisins de l'UE, voir Apap J., Tchorbadjiyska A., « What about the neighbors? Impact of Schengen along the EU's external borders », CEPS Working Document n²10, 2004.

43. Voir Bigo D., « The European internal security field: stakes and rivalries in a newly developing area of police intervention ", in Anderson M., Den Boer M. (eds.), Policing Across 
tat d'actes volontaires d'acteurs individuels ayant des intérêts prédéterminés et un ensemble de contraintes matérielles. Dans ce processus, les acteurs peuvent mettre en avant leurs propres calendriers ou programmes, mais ne pourront décider seuls de la direction d'une culture. En ce sens, les acteurs agissent comme des « vecteurs » des assomptions et des pratiques d'une culture, et l'interaction est le moyen par lequel ces éléments sont articulés et transmis.

Qui a « véhiculé » la culture de Schengen ? Et comment ont-ils « diffusé » les principes sous-jacents à la $\mathrm{PEV}$ ? Nous pouvons déduire des discussions précédentes sur la PEV que la Commission et le Conseil constituent les deux forces motrices à l'origine de cette initiative. Comme nous allons brièvement le montrer, ces deux institutions ont également joué un rôle dans la schengenisation de la PEV. Nous ne devons néanmoins pas omettre le fait que le Conseil et la Commission ne sont pas des acteurs unitaires et cohérents. Plusieurs unités au sein de ces institutions ont participé au processus de formulation et d'élaboration de la PEV (nous pourrions dire la même chose des initiatives menées par l'UE) - parfois en concurrence les unes avec les autres. Parmi ces unités, les directions générales Relations extérieures (DG Relex), Elargissement et Justice, Liberté et Sécurité (DG JLS) de la Commission ; et de l'autre côté les différentes délégations nationales des Etats-membres, agissant avec le Conseil de l'Europe et le Secrétariat général du Conseil. C'est par leur interaction - et non par celle de la Commission ou du Conseil pris en tant qu'entités que cette schengenisation a été rendue possible, et comme nous allons le voir, le Conseil et la Commission ont servi de « vecteurs » de la culture Schengen.

Le Conseil de l'Europe, et plus particulièrement les délégations nationales qui y sont représentées, sont plus « accoutumés » à Schengen que ne le sont les autres institutions ${ }^{44}$. Schengen était une initiative intergouvernementale et, à la fin des années 1990, la plupart des Etats-membres avaient pris part à ses activités. Avec l'incorporation des acquis Schengen dans l'UE, ce régime est entré dans le cadre officiel de la Communauté. Les Etats-membres ont ainsi apporté leurs expériences du régime au sein du Conseil, ce qui s'explique par le rôle crucial joué par le Conseil dans le domaine JAI. En plus des responsabilités partagées avec la Commission dans la plupart des questions liées au domaine JAI, le Conseil avait un contrôle exclusif sur des dossiers sensibles tels que la coopération policière, et servait de point de référence pour la plupart des questions d'affaires étrangères impliquant des questions JAI. La transmission de savoirs et de mémoire institutionnels a été par ailleurs facilitée avec l'arrivée du personnel Schengen au sein du Secrétariat du Conseil lorsque Schengen a été intégré dans l'UE ${ }^{45}$. Ce transfert a renforcé les affinités particulières entre certains Etats-

National Boundaries, London, Pinter Publications, 1994.

44 . Voir Den Boer M., Wallace W., « Justice and Home Affairs », in Wallace H., Wallace W. (eds.), Policy-Making in the European Union, Oxford, Oxford University Press, $3^{\mathrm{e}}$ éd., 2000.

45 . Den Boer M., Corrado L., " For the record or off the record: comments about the incorporation of Schengen into the EU », European Journal of Migration and Law, vol. 1, n4, 1999, p. 413. 
membres et le Secrétariat du Conseil. Mais en dépit de ses références communautaires, le Secrétariat est traditionnellement plus proche des Etats-membres en termes de culture institutionnelle, et donc plus sensible à leurs préoccupations « intergouvernementales » ${ }^{46}$. Cette compréhension mutuelle a, à son tour, facilité l'absorption par le Conseil de l'esprit sécuritaire de Schengen. Cet esprit était clairement présent dans certaines des principales initiatives menées par le Conseil depuis la signature du traité d'Amsterdam, allant du plan d'action conçu lors du sommet de Tampere en 1999 (le premier entièrement dévoué aux questions JAI) au programme récent de la Haye établissant les activités du champ JAI sur le long terme ${ }^{47}$. La sécurité, le contrôle aux frontières et la lutte contre toutes sortes de trafics illicites sont des thèmes récurrents dans ces documents et sont de plus en plus placés au cœur des politiques étrangères européennes.

La Commission européenne a connu une expérience bien plus limitée de Schengen et plus généralement des questions JAI ${ }^{48}$. Cette institution n'avait participé à ce régime qu'en tant qu'observatrice, jusqu'à ce que Schengen soit incorporé à l'UE. Par ailleurs, malgré la création d'un pilier JAI au début des années 1990, son rôle dans ce domaine est resté modeste. La signature du traité d'Amsterdam a accru le pouvoir de la Commission et certains éléments JAI (comme le contrôle des frontières, les politiques d'asile et les politiques migratoires), qui étaient auparavant de la responsabilité seule du Conseil et des Etats-membres, ont intégré ses domaines de compétence. La Commission a obtenu le droit d'initier des propositions dans ces domaines bien que ses compétences fussent partagées avec le Conseil pour une période transitoire de cinq ans, jusqu'en 2004.

Lorsque la PEV a été conçue, le Conseil jouait encore le rôle principal car la Commission n'avait eu qu'une expérience minime des questions JAI (une DG spéciale a été mise en place en 1999, mais jusqu'à cette date, seul un petit groupe de travail était en charge de ces questions au sein de la Commission) et partageait les responsabilités avec le Conseil. En effet, la Commission a souvent subi une certaine pression politique de la part d'Etats-membres afin qu'elle suive un programme plus sécuritaire. Dans de nombreux cas, la Commission a soutenu des Etats-membres puissants au sein du Conseil et a interprété certaines règles de manière restrictive, comme celles relatives au droit d'asile. Cela s'explique en partie du fait de la socialisation (en participant aux pratiques communes, la Commission a absorbé les principes de cette culture, faisant ainsi les premiers pas vers son internalisation), et

45 . Den Boer M., Corrado L., « For the record or off the record: comments about the incorporation of Schengen into the EU », European Journal of Migration and Law, vol. 1, n 4, 1999, p. 413.

46. Il convient de rappeler que le rôle institutionnel du Secrétariat est d'apporter une aide administrative, juridique et politique aux Etats-membres.

47. Conseil de l'Europe, The Hague Programme: Strengthening Freedom, Security and Justice in the European Union, Brussels, 13 décembre 2004, 16054/04 (JAI 559).

48. Uçarer E.M., "From the sidelines to center stage: sidekick no more? The European Commission in Justice and Home Affairs ", European Integration online Papers, n5, 2001. 
partiellement du fait d'un certain pragmatisme (elle est venue à la conclusion qu'il valait mieux calmer les Etats-membres qu'aller dans le sens d'un programme alternatif qui aurait de toute façon très certainement été bloqué) ${ }^{49}$.

L'histoire de la PEV est également caractérisée par cette relation dialectique entre le Conseil et la Commission et la schengenisation du discours sur les questions JAI qui en a résulté. L’orientation sécuritaire de la PEV était déjà visible dès les débuts de l'initiative, comme l'atteste la lettre de Straw à la présidence espagnole. Mais lorsque la Commission a commencé à travailler sur le dossier PEV, il n'y avait aucun détail sur le contenu et l'organisation de cette initiative, en particulier sur qui devrait en avoir la charge, en dehors de quelques lignes directrices. Le groupe de travail «Europe élargie » n’a été instauré qu'en juillet 2003. La DG Relex a fait la majorité du travail tandis que d'autres DG jouèrent des rôles importants dans l'élaboration de la politique. Les tentatives de la part des membres de la DG Elargissement (en particulier son directeur Verheugen) d'influencer la PEV et les luttes politiques avec les collègues de la DG Relex sont connues ${ }^{50}$. La DG Justice, Liberté et Sécurité (JLS) a exercé une influence tout aussi importante - bien que moins visible - au sein de la Commission ${ }^{51}$. La JLS est une des «line DG» invitée à participer à l'élaboration de la politique grâce à ses compétences et son expertise. Au fur et à mesure que la PEV s'est précisée, la DG JLS a exigé de plus en plus de place dans le planning et son influence a grandi. De par le sujet qu'elle aborde et ses nombreux contacts avec les ministères de l'Intérieur des Etats-membres, cette DG tend à prôner une orientation plus sécuritaire que les autres DG et a ouvertement demandé à ce que des sujets tels que le droit d'asile, l'immigration clandestine et le trafic soient placés en priorité dans le programme de la PEV. Cette position est entrée en conflit avec celle de la DG Relex qui considérait que ces questions devaient être considérées comme des éléments parmi d'autres dans la politique globale ${ }^{52}$. La dimension sécuritaire de la PEV a largement été mise en avant dans la proposition de la Commission et dans les plans d'action à l'encontre des pays voisins du fait de l'activisme de la DG JLS.

La poussée décisive vers une perspective sécuritaire de la PEV n’est pas venue de la Commission, mais de son homologue institutionnel, le Conseil. Depuis le lancement de la politique en 2002, le Conseil ne s'était pas directement impliqué, laissant la Commission se charger des détails. A la suite de la publication de la Communication de 2003 sur l'Europe élargie, le Conseil a

49. Voir Bigo D., Guild E., "De Tampere à Séville : bilan de la sécurité européenne ", Cultures $\mathcal{E}$ Conflits, $\mathrm{n}^{\circ} 45 / 46,2002$, pp. 5-18.

50. Voir Goujon A., «L'Europe élargie en quête d'identité : légitimation et politisation de la politique européenne de voisinage », Politique européenne, n¹5, 2005, pp. 137-163.

51 . Jeandesboz J., "Alternative narratives of the European Neighbourhood policy: elements for a "genesis" of the ENP ", article présenté lors du séminaire "Perspectives on the European Neighbourhood Policy », CEPS, Bruxelles, 21-22 avril 2006, pp. 34-35.

52 . Ibid., p. 25. 
réévalué son rôle et exercé une pression politique grandissante sur la Commission ${ }^{53}$. La raison principale de ce changement d'attitude du Conseil vient de la croyance selon laquelle la Commission outrepassait ses rôles, notamment lorsqu'elle entamait des négociations directes avec certains voisins dans le cadre des plans d'action. Le Conseil a donc décidé de superviser davantage les activités de la Commission et d'avoir un droit de regard sur le contenu de la PEV, en particulier lorsque le sujet était politique ou qu'il relevait de questions de politique étrangère. Ce changement d'attitude du Conseil s'est exprimé dans sa décision de prendre la responsabilité de désigner et d'ouvrir les négociations officielles avec les voisins.

L'effet de cette prise de pouvoir du Conseil est visible dans le Document d'orientation de 2004. Comme nous l'avons vu, la Commission a éliminé certains des éléments les plus progressistes de la Communication sur l'Europe élargie (telle que la possibilité de bénéficier des « quatre libertés » de l'UE). Quelles que soient les raisons de cette position (pragmatisme, socialisation, ou des pressions directes du Conseil), la Commission a, ce faisant, non seulement reproduit certains éléments clefs de Schengen, mais a aussi aidé, volontairement ou non, à légitimer davantage le discours sous-jacent. Cela a également créé certaines tensions et contradictions au sein de la PEV, comme nous l'avons vu plus tôt. Les éléments sécuritaires de Schengen se heurtent en fait à d'autres objectifs de la PEV, et particulièrement ceux de prospérité et de relations amicales avec les voisins. En ce sens, la PEV peut être perçue comme la dernière «victime » de la propagation de la culture Schengen de la sécurité dans de nouveaux domaines de politique européenne.

\section{Une PEV schengenisée et ses perspectives}

Nous avons tenté de mettre en avant les dimensions sécuritaires et eurocentrées de la PEV, une initiative lancée par l'UE pour reformuler ses relations avec son "proche étranger» après la dernière vague d'élargissement. L'examen critique du rôle de la sécurité dans la PEV montre que cet exemple de politique étrangère civile de l'UE est plus controversé que veulent bien le laisser entendre ses défenseurs. D’un côté, l'exigence de répondre à des menaces existantes ou potentielles qui pourraient mettre en danger la paix et la stabilité dans la région a eu pour conséquence de limiter les motivations et incitations offertes aux voisins. D'un autre côté, la manière dont ces questions de sécurité ont été formulées et intégrées dans la PEV révèle un euro-centrisme clair. Partant d'un cadre d'analyse sociologique, nous avons ensuite tenté de montrer que ces éléments viennent d'une évolution de la « culture Schengen de la sécurité » en mettant en avant les rôles respectifs que les principaux

53. Le Haut Représentant de l'UE est également devenu plus actif. C'est en fait à cette période (décembre 2003) que Javier Solana a officiellement présenté la stratégie européenne de sécurité. Comme nous l'avons vu, ce document contient une section dédiée aux questions de voisinage. 
défenseurs du projet (la Commission et le Conseil) ont joué dans l'inclusion de ses principes dans la PEV.

Quelles sont les conséquences d'une telle ligne argumentative pour l'avenir de la PEV ? Puisque ses éléments actuels résultent d'un processus en cours de consolidation de la culture Schengen de la sécurité, il semble difficile de pouvoir changer son cours pour le moment. Mais cela ne signifie pas que l'on ne puisse remettre en cause cette culture. Une culture de la sécurité n'est ni statique ni immuable, et son évolution résulte d'une interaction sociale entre les acteurs qui y participent, ce qui permet un certain changement dans certaines circonstances. Dans le contexte de la PEV, une transformation potentielle peut venir des voisins eux-mêmes. Jusqu'à présent, les pays de l'Europe de l'Est et du Sud-Méditerranée impliqués dans la politique ont accepté la schengenisation de la PEV, en suivant - quoiqu'à contre-cœur - les exigences de l'UE, dans l'espoir de pouvoir profiter économiquement de ce rapprochement avec l'UE. Des signes d'irritation peuvent déjà se faire sentir chez certains voisins. A mesure que la $\mathrm{PEV}$ prend forme et que les premiers plans d'action sont appliqués, certains gouvernements appellent à plus de concessions de la part de l'UE, et résistent aux politiques impopulaires que cette dernière tente de leur faire imposer à leurs populations. L'UE a, de son côté, reconnu certaines lacunes de l'initiative telle qu'elle a été formulée à l'origine, et s'est engagée à rendre les incitations offertes aux voisins plus acceptables qu'elles ne l'étaient ${ }^{54}$.

Mais en dépit de ces efforts, il reste peu de possibilités de changer l'organisation de la PEV et ses priorités. Les événements du 11 septembre aux EtatsUnis et les attaques terroristes récentes sur le sol européen ont encore renforcé le discours sécuritaire dominant les politiques européennes et a rendu très ardue toute tentative de les dépasser 55 . Dans ce contexte, la situation délicate, et fâcheuse, de la PEV risque fort de durer.

54. Voir les discussions sur les «forces et faiblesses» de la PEV dans la communication de la Commission relative au "Renforcement de la politique européenne de voisinage », COM (2006) 726 final, 2006, p. 3 et suivantes. La Commission exige notamment que des actions urgentes soient effectuées dans le domaine des visas : «Il est vrai, pourtant, que nos politiques et pratiques existantes en matière de visas sont souvent sources de difficultés et d'obstacles réels pour les déplacements effectués pour des motifs légitimes. Les longues files d'attente devant les consulats de l'Union européenne sont la preuve flagrante de l'existence de barrières à l'entrée dans l'Union». (Ibid., p. 6).

55. Pour une analyse de l'impact du 11 septembre sur le JAI de l'UE, voir Guild E., «International terrorism and EU immigration, asylum and borders policy: the unexpected victims of 11 September 2001 », European Foreign Affairs Review n8, 2003. 